\title{
A steady-state supersonic downflow in the transition region above a sunspot umbra ${ }^{\star}$
}

\author{
Thomas Straus ${ }^{1}$, Bernhard Fleck ${ }^{2}$, and Vincenzo Andretta ${ }^{1}$ \\ 1 INAF/Osservatorio Astronomico di Capodimonte, via Moiariello 16, 80131 Napoli, Italy \\ e-mail: straus@oacn.inaf.it \\ 2 ESA Science Operations Department, c/o NASA/GSFC, Greenbelt, MD 20771, USA
}

Received 4 February 2015 / Accepted 15 July 2015

ABSTRACT

\begin{abstract}
We investigate a small-scale $\left(\sim 1.5 \mathrm{Mm}\right.$ along the slit), supersonic downflow of about $90 \mathrm{~km} \mathrm{~s}^{-1}$ in the transition region above the lightbridged sunspot umbra in AR 11836. The observations were obtained with the Interface Region Spectrograph (IRIS) on 2013 September 2 from 16:40 to 17:59 UT. The downflow shows up as redshifted "satellite" lines of the Si IV and O IV transition region lines and is remarkably steady over the observing period of nearly $80 \mathrm{~min}$. The downflow is not visible in the chromospheric lines, which only show an intensity enhancement at the location of the downflow. The density inferred from the line ratio of the redshifted satellites of the OIV lines $\left(N_{\mathrm{e}}=10^{10.6 \pm 0.25} \mathrm{~cm}^{-3}\right)$ is only a factor 2 smaller than the one inferred from the main components $\left(N_{\mathrm{e}}=\right.$ $\left.10^{10.95 \pm 0.20} \mathrm{~cm}^{-3}\right)$. Consequently, this implies a substantial mass flux $\left(\sim 5 \times 10^{-7} \mathrm{~g} \mathrm{~cm}^{-2} \mathrm{~s}^{-1}\right)$, which would evacuate the overlying corona on timescales close to $10 \mathrm{~s}$. We interpret these findings as evidence of a stationary termination shock of a supersonic siphon flow in a cool loop that is rooted in the central umbra of the spot.
\end{abstract}

Key words. sunspots - Sun: atmosphere - Sun: oscillations - Sun: transition region

\section{Introduction}

Sunspots have been an area of intense research ever since Hale's (1908) discovery of strong magnetic fields in these structures (see reviews by, e.g. Borrero \& Ichimoto 2011; Rempel \& Schlichenmaier 2011). Here we investigate a particular sunspot phenomenon: a small-scale ( $\sim 1.5 \mathrm{Mm}$ along the slit), supersonic, relatively stable downflow of about $90 \mathrm{~km} \mathrm{~s}^{-1}$ in the transition region above a sunspot umbra, which was first noticed by Tian et al. (2014a) in the same data set.

A similar phenomenon was first seen in data obtained during the 1975 July 21 flight of the NRL High Resolution Telescope and Spectrograph (HRTS) by Dere (1982) and Nicolas et al. (1982), and later in SOHO/SUMER observations by Brynildsen et al. (2001, 2004). Since HRTS provided only a snapshot, no inferences could be made about the temporal variability or stability of this feature. Brynildsen et al. (2001), on the other hand, did have temporal information in the SUMER sit-and-stare time series they studied. In the 20 min time series they presented, they found line profiles that were well represented by two Gaussian line components in the N V $1242 \AA$ and O V $629 \AA$ lines, with the main component showing prominent 3 min oscillations and the redshifted component showing nearly the same high velocity $\left(\sim 90 \mathrm{~km} \mathrm{~s}^{-1}\right.$ in one data set) without oscillations in both emission lines, for which they coined the phrase "dual flow". However, they did not discuss the origin of these dual flows in any detail. Brynildsen et al. (2004) extended their earlier work and found dual flows (i.e. line profiles in which two distinct velocities could be observed within the same resolution element) in five out of the 12 sunspots they studied.

$\star$ The movie is available in electronic form at http: //www . aanda.org
There have been numerous reports of supersonic downflows above sunspots following the initial HRTS publications by Dere (1982) and Nicolas et al. (1982). Most of them are based on observations with HRTS I and II and HRTS on Spacelab 2 (e.g. Brekke et al. 1990, 1991a,b; Kjeldseth-Moe et al. 1988, 1993), and they often showed multiple flow components within the $1^{\prime \prime} \times 1^{\prime \prime}$ resolution element. It is not clear, however, whether all these flows are akin to the dual flows of Brynildsen et al. (2001). Furthermore, Gurman (1993) only measured weak, mostly subsonic, downflows when analysing observations of eight sunspots with UVSP on the Solar Maximum Mission (SMM). He did note an interesting aspect where the sunspots of his work differed from those observed by HRTS: the latter ones all had obvious lightbridges, while only one of the eight he observed with UVSP did. Kjeldseth-Moe et al. (1988) also speculated that the flows, which they found to have a typical size of $5^{\prime \prime}$, might be associated with structures such as lightbridges.

In addition to the two Brynildsen et al. papers cited above, there have been several other studies of transition region downflows above sunspots based on data from SUMER and CDS on SOHO (e.g. Brynildsen et al. 1998; Brosius \& Landi 2005). We believe these downflows are different, however, from the phenomenon that we are describing in this paper. They were not as strong, but were mostly subsonic or, in any case, below $35 \mathrm{~km} \mathrm{~s}^{-1}$, and more extended, often covering a large portion of the penumbra.

Supersonic downflows in the transition region above a sunspot have also recently been detected by Kleint et al. (2014) with the Interface Region Spectrograph (IRIS; De Pontieu et al. 2014). These authors observe bursts of extremely broad line profiles in Mg II h and k, C II $1336 \AA$, Si IV $1394 \AA$, and $1403 \AA$, suggesting supersonic downflows of up to $200 \mathrm{~km} \mathrm{~s}^{-1}$ and weaker upflows that are associated with small-scale brightenings in 
sunspot umbrae and penumbrae. The broad line profiles do not have distinct velocity components such as the multiple flows observed by Dere (1982), Nicolas et al. (1982), Kjeldseth-Moe et al. (1993), Brynildsen et al. (2001, 2004), and they appear rather intermittently in short bursts lasting about $20 \mathrm{~s}$. These characteristics are very different from the rather steady, mono-chromatic downflow discussed in this paper, which reveals itself as Gaussian satellite lines in the spectra of Si IV and O IV and which is well separated from the main components. Interestingly, such a well-separated component is also visible in the $\lambda$-t-plots displayed in Fig. 3 of Kleint et al. (2014) at about $100 \mathrm{~km} \mathrm{~s}^{-1}$ redshift, but this is not discussed in the paper.

In Sect. 2 we describe the observations. The analysis and results are presented in Sect. 3 and discussed in Sect. 4, before concluding in Sect. 5.

\section{Observations}

For this study we used the same data set as Tian et al. (2014a) for their study of the propagation behaviour of shock waves in a sunspot. The data were obtained with the IRIS in sit-andstare mode on 2013 September 2 from 16:39:35 to 17:58:45 UT. The target was the sunspot in AR 11836 near disk centre at $x=106^{\prime \prime}, y=58^{\prime \prime}$ (coordinates of the centre of the slit at the beginning of the observations). Slit length and width were $68^{\prime \prime}$ and 0 '. 166 , respectively. The pixel size along the slit is $0{ }^{\prime} 166$. The data were acquired by binning the far-UV (FUV) data in the wavelength direction by a factor of two, and thus have a dispersion of $\sim 25 \mathrm{~m} \AA$ per pixel (about $5 \mathrm{~km} \mathrm{~s}^{-1}$ at $1400 \AA$ ) in both the NUV and FUV channels. The cadence of the spectral data was three seconds, with exposure times of two seconds. The observations comprise nine spectral windows: C II $1336 \AA$, Fe XII 1349 A, Cl I 1352 A, O I $1356 \AA$, Si IV $1394 \AA$, Si IV $1403 \AA$, NUV at $2786 \AA$ and $2831 \AA$, and Mg II h, and $\mathrm{k} 2796 \AA$. The spectral window containing the Si IV $1403 \AA$ line also includes the O IV $1400 \AA, 1401 \AA$, and $1405 \AA$ lines, the last one blended with a S IV line. In parallel to the spectral data, slit-jaw images were taken in three filters $(2796 \AA$, $1400 \AA$, and $1330 \AA$ ) at a 12 s cadence. The pixel size of the slit-jaw images is 0 ' $^{\prime} 166 \times 0$.' 166 . We used Level-2 data from the IRIS archive at Lockheed-Martin Solar and Astrophysics Laboratory (LMSAL), i.e. data that have already been corrected for dark current, flat-field, and geometrical distortions. Below, we also discuss the line ratios of the Si IV doublet and the O IV density sensitive lines. For that purpose we applied the current estimate of the wavelength-dependent effective area, as provided by the Solarsoft routine iris_get_response.pro, using version 3 of the calibration that was released on 2015 April 1.

For context, as well as to gain some information about the higher temperature plasma and the magnetic field structure, we complemented the IRIS observations with data from the Atmospheric Imaging Assembly (AIA; Lemen et al. 2012) and the Helioseismic and Magnetic Imager (HMI; Scherrer et al. 2012) on the Solar Dynamics Observatory (SDO; Pesnell et al. 2012). To allow a comparison with the IRIS observations, the AIA and HMI data were re-mapped to the IRIS data, both spatially and temporally, using metadata in the FITS headers and the map routines of SolarSoft. No additional roll angle correction has been applied. A secondary step in SDO-IRIS cross-alignment was achieved by comparing the HMI whitelight images and the near continuum data from the $2831 \AA$ spectral window of IRIS (wavelength index 15). The different IRIS spectral windows have been cross-aligned using the fiducial lines. No further inter-instrument alignment between HMI and the different AIA channels was applied. In a final step, temporal jitter and drift of IRIS along the slit was removed from the spectral data by cross-correlation techniques applied to the near continuum intensity in the $2831 \AA$ spectral window. The totally accrued drift during the observations was less than 3.5 IRIS pixels, or the equivalent of one HMI pixel (0.'5). Pointing excursions of IRIS perpendicular to the slit cannot be corrected. All things considered, we believe that the cross-alignment between the IRIS and SDO data is accurate to within one HMI pixel.

\section{Observational results}

We start with a summary of the characteristics of the observed supersonic downflow. The various findings are discussed in more detail below. Throughout this paper, we adhere to the convention that positive velocities represent blueshifts. The downflow

- shows up as redshifted satellite lines of the SiIV and O IV transition region lines, separated from the main components by about $90 \mathrm{~km} \mathrm{~s}^{-1}$ (Figs. 1-4);

- is very localised and small-scale $(\sim 1.5 \mathrm{Mm}$ along the slit, see Fig. 1);

- occurs in the centre of a sunspot umbra that has one prominent and two smaller lightbridges (Fig. 1);

- is a very steady-state flow, both in line intensity and in velocity (Fig. 4), lasting at least as long as the observations with surprisingly little variation for the transition region, which is usually highly dynamic (see Kleint et al. 2014);

- does not participate in the 3 min shock wave dynamics that dominate the main components (Fig. 4) and shows only very weak, anti-correlated intensity signatures (Fig. 6);

- is comparatively more pronounced (at about $60 \% \pm 20 \%$ of the main component) in the O IV lines than in the Si IV doublet $(\sim 20 \% \pm 10 \%$ of the main component);

- has a density of about $4 \times 10^{10} \mathrm{~cm}^{-3}$, with little temporal and spatial variations, and accordingly, a mass flux of $\sim 5 \times$ $10^{-7} \mathrm{~g} \mathrm{~cm}^{-2} \mathrm{~s}^{-1}$

- has a density a factor $\sim 2$ lower than the one inferred from the main component (Fig. 5);

- is visible only in the Si IV and OIV transition region lines with no velocity signature in the chromospheric lines $\mathrm{Mg}$ II $\mathrm{h}$ and $\mathrm{k}$ or C II (Fig. 2);

- is co-spatial with an increased intensity in those same chromospheric lines, suggesting related enhanced heating (Fig. 2);

- is not characterised by distinctly peculiar line profiles; in particular, the profiles of the Si IV and OIV satellite lines are nearly Gaussian with widths that are about the same as those of the main components, although the width of the Si IV satellite lines tends to be about $40 \%$ larger than the main component with large uncertainties (Fig. 3).

\subsection{Context of observation}

Figure 1 provides an overview of the region studied in this paper. The upper left shows a HMI full disk magnetogram, and the upper right, a cut-out HMI magnetogram and AIA $171 \AA$ intensity image of AR 11836. The lower left shows an even more zoomedin HMI intensity image of the central sunspot of AR 11836. The position of the IRIS slit is indicated $(x=0)$. The lower right shows a 1-min average of the spectral window containing the Si IV $1403 \AA$ line. The red symbols indicate the region (about $2.8 \mathrm{Mm}$ wide) in the umbra where the Si IV and O IV lines show 

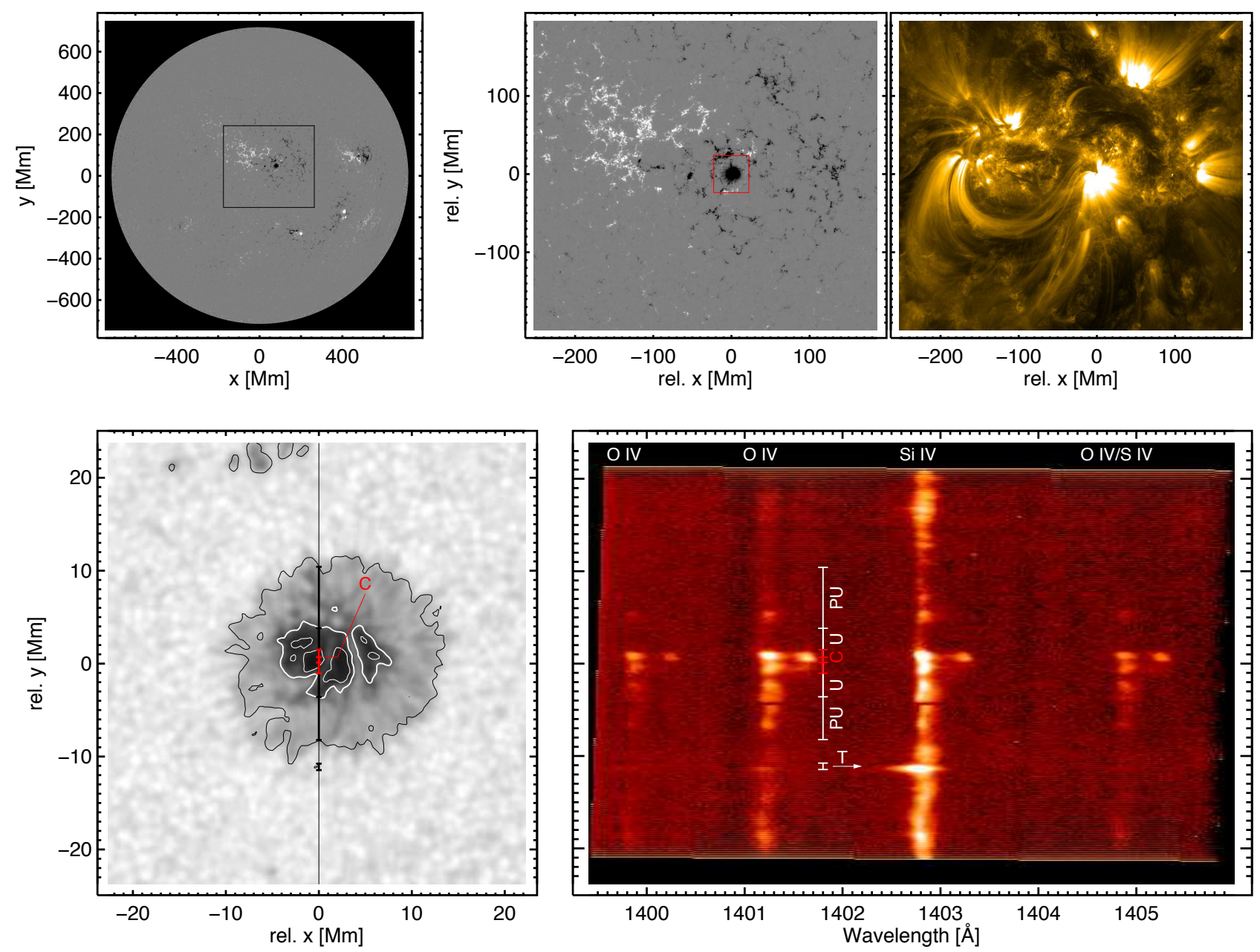

Fig. 1. Upper panels (context images of AR 11836): full disk (left) and cut-out (middle) HMI magnetogram and corresponding AIA $171 \AA$ Amage (right). Lower panels: zoom of HMI continuum image on the area marked by the red box in the upper panel, with the IRIS slit marked at $x=0$ (left), and the corresponding spectra in the Si IV $1403 \AA$ window averaged over 1 min (right panel). All data were taken on 2013 Sep. 2, 17:14 UT. Regions where the slit crosses the penumbra (PU) and umbra (U) are marked. The central part of the umbra, where the Si IV and O IV lines show satellites, is indicated in red. In the northern part, where the satellites are strongest, the centre of this region is labelled C and used as the reference point of the satellite feature in this report. The prominent upflow, labelled T, is due to a short-lived transient.

satellites. In the northern part, the satellites are strongest, with the size of this northern part about $1.5 \mathrm{Mm}$. The centre of this region is labelled $\mathrm{C}$ and used as the reference point of the satellite feature in this report.

The same data set has been studied and described by Tian et al. (2014a) and Yurchyshyn et al. (2015), who investigate the strong 3-min umbral oscillations found in this spot. It is interesting to note that Point $\mathrm{C}$ seems to coincide with both the apparent source of the umbral running waves studied by Yurchyshyn et al. (2015) and with the location of the most intense magnetic field in the sunspot.

As can be seen in the HMI images, the spot of AR 11836 is an isolated, small, round spot of leading polarity. There is no following spot, only active region plage. The spot is surrounded by a large area with a network field of the same polarity. Opposite polarity can be found in only a few locations south and north east of the spot. The loops visible in AIA $171 \AA$ are inclined and connect the spot to plage of opposite polarity following the spot. The loops seem to be anchored in the dark north west quadrant, whereas the rest of the sunspot displays a bright plume in the AIA $171 \AA$ images.

The sunspot shows an irregular umbra, split into two pieces by a prominent lightbridge in the western part. The bigger, eastern part is split further into three pieces by two smaller, less prominent lightbridges. According to Gurman (1993) it might be important for the presence of a supersonic downflow that this sunspot has a lightbridge. The slit of the IRIS spectrograph crosses the eastern part centrally in the north-south direction.

\subsection{Average properties}

The Si IV and OIV lines form at approximately $70000 \mathrm{~K}$ and $150000 \mathrm{~K}$, respectively. In both lines, the supersonic downflow is stable enough to be prominent in the spectra averaged over the whole 80 min of the observations (see Fig. 2). The strong northern satellite region is the darkest part in the pseudo-continuum between the $\mathrm{Mg}$ II $\mathrm{h}$ and $\mathrm{k}$ lines and shows, at the same time, the brightest intensity in all emission lines. There is no hint of any redshifted components in the chromospheric spectral bands, in particular also not in the strong Mg II $\mathrm{h}$ and $\mathrm{k}$ and C II $1336 \AA$ lines.

To proceed with a quantitative analysis of the line profiles, we determined the main line parameters, namely the peak value, $I_{\max }$, its position, $\lambda_{\max }$, and the full width at half maximum (FWHM) by means of a parabolic fit to the central three pixels of each profile. Since the core of the Si IV and O IV lines 


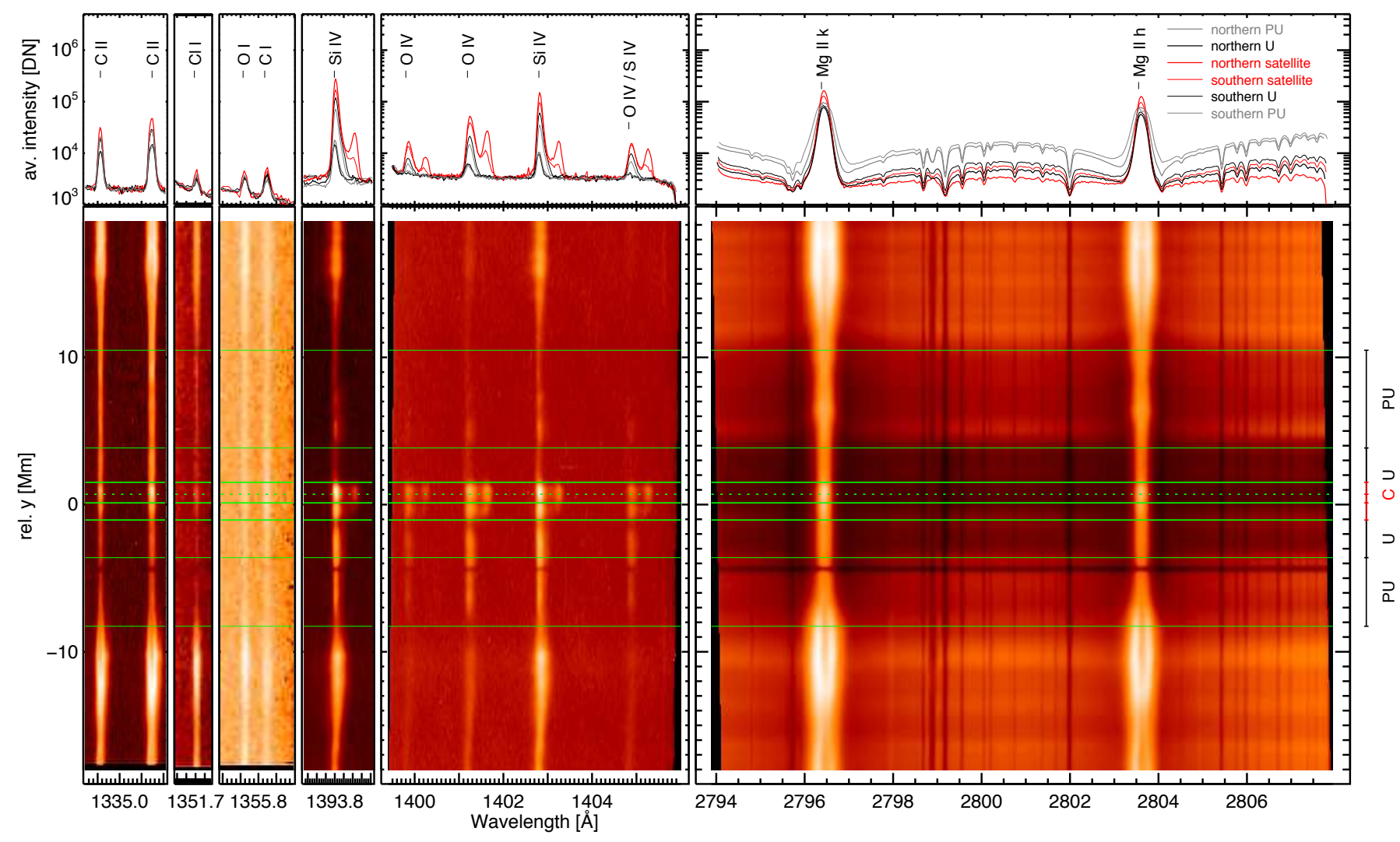

Fig. 2. Spectra averaged over the whole time series as a function of wavelength and position along the slit (lower panels, logarithmic colour scale) and further over the different regions (upper panels). The labels of different regions, in the lower row, correspond to those given in Fig. 1.

is only three or four pixels wide in this data set, a fit to a larger number of pixels is normally not required. At the extrema of shock maneuvres, double-peaked profiles may appear, in which case the fit procedure then identifies the strongest peak. If needed, the total line intensity, $I_{\text {tot }}$, is computed, as in the case of Gaussian profiles, as the peak value times the line width: $I_{\mathrm{tot}}=$ $0.5 \sqrt{(} \pi / \log 2) \times I_{\max } \times F W H M=1.064467 \times I_{\max } \times F W H M$. We have compared this procedure with a more standard Gaussian fit, verifying that the agreement is excellent, especially for the more prominent lines. The Gaussian fit procedure, however, tends to fail more often in the case of weak profiles. The double peaks at the extrema of shock maneuvres are also less clearly identified. Finally, Gaussian fits assume symmetric profiles, while parabolic fits do not make this assumption.

The average properties of the line parameters thus estimated were then determined from the histograms computed over 13 pixels centred on Point $\mathrm{C}$ along the slit, in other words, the region within the two marks nearest to Point $\mathrm{C}$ in Fig. 1 (the northern satellite region). A Gaussian fit to the peak of the histogram yields both the average value and the standard deviation. We estimated the averages over the full time span and for the first $30 \mathrm{~min}$ of the observing run only. We computed the average over the first $30 \mathrm{~min}$ because, after that time, the redshifted components become fainter. Moreover, after about $30 \mathrm{~min}$, the mean position along the slit of the downdraft region starts drifting south with respect to Point $\mathrm{C}$ by several pixels. The average values do not change much in either case: for the Si IV line the mean shift between the two components is $91 \pm 8 \mathrm{~km} \mathrm{~s}^{-1}$, while the average value for the first $30 \mathrm{~min}$ is $94 \pm 6 \mathrm{~km} \mathrm{~s}^{-1}$; the corresponding values for the O IV lines are $82 \pm 8 \mathrm{~km} \mathrm{~s}^{-1}$, while the average for the first $30 \mathrm{~min}$ is $84 \pm 6 \mathrm{~km} \mathrm{~s}^{-1}$. In the following, we always refer to averages computed for the first $30 \mathrm{~min}$, unless explicitly stated otherwise.

The satellite lines are most pronounced in O IV, with the total intensity being on average approximately $60 \pm 15 \%$ of the main component. The average Si IV satellite line is about $20 \pm 10 \%$ of the total intensity of the main component. Variations in the relative intensity of the satellite to the main component in different O IV lines can be interpreted as resulting from density variations (see discussion of Sect. 3.3.2). Since we do not have estimates of plasma density in the region where the Si IV lines form, we are unable to offer a simple explanation of the different behaviour of this ratio between satellite and main component to the O IV lines. This piece of information may be useful for constraining more detailed models of this phenomenon. However, we note that Nicolas et al. (1982) used a larger set of lines to already estimate that the shape of the differential emission measure of the supersonic downflows is drastically different from the curve corresponding to the surrounding umbra. In particular, the differential emission measure they found in the downflows (their Fig. 8) is similar to the one they found in the sunspot umbra for $\log T>5$, when it continues to drop towards lower temperatures, as opposed to the increase after that turn-around point seen in the umbra. This implies that the intensity of the O IV in downflows should be comparable to the one observed in the surrounding umbra, while the Si IV lines intensities should be strongly suppressed, as observed.

We performed a similar analysis of the southern part of the satellite region. There the satellite lines are weaker than the main component: the OIV satellite lines are on average only about $10 \%$ of the main component, while the SiIV satellites are $5 \%$ or less. However, the velocity shifts remain as stable 
T. Straus et al.: A steady-state supersonic downflow in the TR above a sunspot umbra

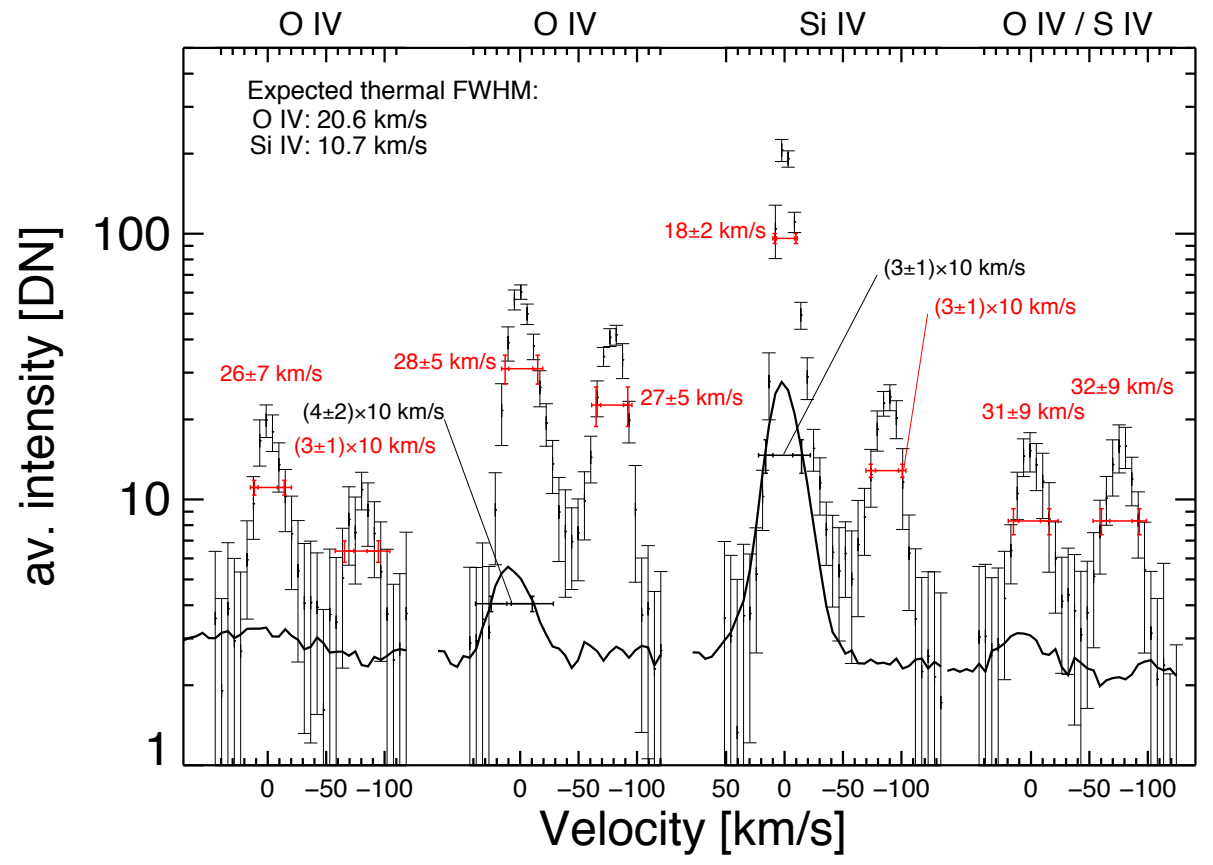

Fig. 3. Symbols: 1-min average line profiles at Point $\mathrm{C}$ with error bars (standard deviation of the 20 individual measurements), taken 5 min before the reference spectrum in Fig. 1 (17:09 UT). At this time, the satellite lines in O IV are particularly strong. Solid line: average spectrum outside the spot. The main and satellite lines all have similar line widths $(F W H M \sim$ $30 \mathrm{~km} \mathrm{~s}^{-1}$ ), with the exception of Si IV $1403 \AA$, which has a significantly narrower main component. The satellites are less pronounced in Si IV than in O IV. and comparable in magnitude to the values measured around Point C, although slightly smaller on average: $\sim 70 \mathrm{~km} \mathrm{~s}^{-1}$ for the O IV lines and $\sim 80 \mathrm{~km} \mathrm{~s}^{-1}$ for the Si IV lines.

\subsection{Plasma diagnostics}

For the rest of this section, except when noted otherwise, we analyse line profiles, which were calibrated according to the procedure described in IRIS Technical Notes \#1 and \#24 $4^{1}$, and adopted the most recent effective areas for the IRIS spectrograph as provided by the SolarSoft routine iris_get_response .pro (version 3, 2015 April 1).

\subsubsection{Line optical thickness}

In the plage regions outside the sunspot, the profiles of the $\mathrm{C}$ II lines at $1334.5 \AA$ and $1335.7 \AA$ display an evident selfreversal, a clear signature of a significant optical thickness. Even in the penumbra, the ratio of the total intensities of the two components, in contrast with the optical thin limit of $1: 2$, is around $1: 1.4$, a value that is close to the observed value in quiescent regions (Judge et al. 2003) and to the computed values in the reference quiescent chromosphere by Avrett \& Loeser (2008).

On the other hand, the profiles in the region around Point $\mathrm{C}$ are nearly Gaussian. Nevertheless, the line widths of the C II $1334.5 \AA$ are, on average, larger than those of the C II $1335.7 \AA$ line in the northern satellite region $(1.3 \pm 0.2)$, while at the same time, the ratio of the total line intensities is consistent with the optical thin value $(2.0 \pm 0.3)^{2}$. These two measurements together suggest that the C II doublet in that region is affected by a modest optical thickness that is possibly smaller than in the quiet Sun and that it is most likely being formed in the effectively optically thin regime, where the line profiles become broader before the total line intensity is reduced

\footnotetext{
1 http://iris.lmsal.com/documents.html

2 The $1335.7 \AA$ line is in reality composed of two lines at $1335.71 \AA$ and $1335.66 \AA$; the latter line, however, is much weaker and does not therefore affect significantly the width of the observed profile.
}

by the destruction of photons as a result of collisions in the multiple scattering process. These measurements are consistent with earlier estimates (e.g. Jordan et al. 1978; Bartoe et al. 1979; Tian et al. 2009), suggesting a lower opacity of the chromosphere above sunspot umbrae.

The average line profiles of the Si IV $1393.8 \AA$ and $1402.8 \AA$ lines do not show any sign of self-reversal or of significant departures from Gaussian in the line core in any region crossed by the slit (Fig. 2). We cannot find any clearly non-Gaussian profiles that can be attributed to optical thickness effects in the data we browsed, although we cannot check all the individual profiles in the data set.

A first analysis of the intensity ratio in the northern satellite region around Point " $C$ " of the main components of the two Si IV lines nevertheless shows the mean ratio of the $1394 \AA$ to the $1403 \AA$ to be around 1.4 , a value that is significantly lower than the optical thin line ratio of 2 . While revising this paper, however, an updated, post-launch radiometric calibration of IRIS was released. With this latest calibration, the ratio of the main components of the SiIV 1393.8 and $1402.8 \AA$ lines becomes $1.9 \pm 0.1$, which is much closer to the optically thin ratio. The ratio of the widths of the main component of the Si IV $1402.8 \AA$ line to the $1393.8 \AA$ line is on average $1.04 \pm 0.08$ in the same region, a value close to the value of 1.07 given by Doschek \& Feldman (2004) for the quiet Sun.

If we take the results of the analysis of the $\mathrm{C}$ II lines above as also relevant to the main $\mathrm{Si}$ IV lines, i.e. that the optical thickness in Point $C$ is even smaller than in the average quiet Sun, we can also cite previous studies carried out in quiescent regions. In particular, the analysis of Roussel-Dupre et al. (1979) of the limb brightening of the line intensity of Si IV $1393.8 \AA$ in OSO- 8 data indicates an optical depth of $0.22 \pm 0.16$ in the quiet Sun, in contrast to the much larger optical thickness of the C II $1334.5 \AA$ and $1335.7 \AA$ doublet estimated by Avrett \& Loeser (2008). Moreover, the Si IV lines $1393.8 \AA$ and $1402.8 \AA$ lines are not listed in Table 3 of Doschek \& Feldman (2004) amongst lines with measurable optical thickness in the FUV spectral range as observed by SOHO/SUMER. 

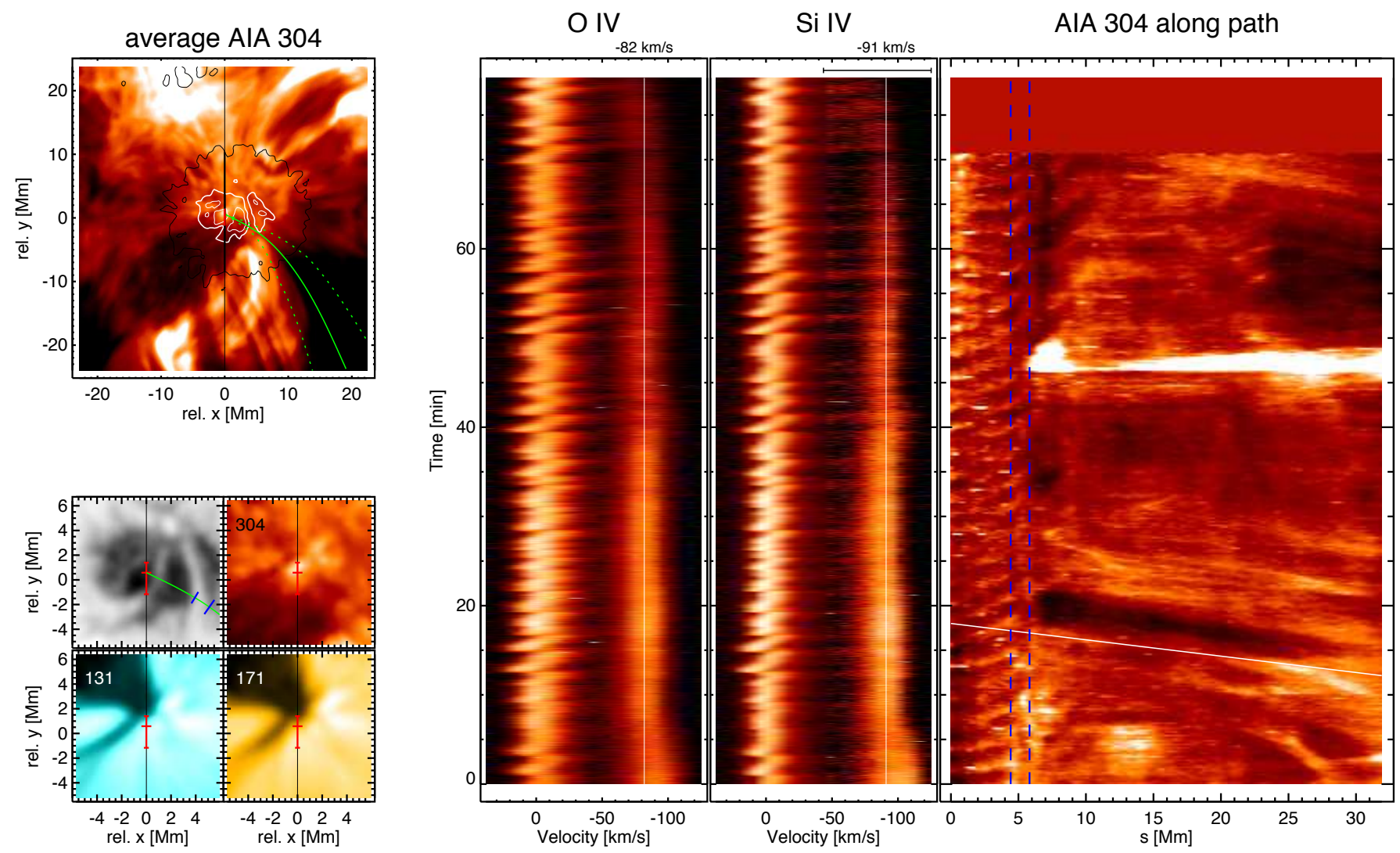

Fig. 4. Upper left-hand panel: temporal average of AIA $304 \AA$ Antensity, with HMI continuum intensity contours from Fig. 1 overlayed. The green solid and dashed lines indicate a path of inflows and the area of integration used for the time-distance plot in the right panel. Lower left panels: temporal average images of HMI continuum intensity and three AIA channels. The red marks indicate the satellite region and Point $\mathrm{C}$ as in Fig. 1. Central panels: temporal evolution of the O IV and Si IV lines averaged over 13 pixels in the northern satellite region centered on Point C. The region of the satellite in Si IV, indicated by the bar on top, has been contrast-enhanced to better show the weak connection to the main component's shock maneuvres. Right panel: relative AIA $304 \AA$ intensity fluctuations, integrated across the path identified by the green lines in the context image in the upper left. The colour table maps variations from $-30 \%$ to $+30 \%$. Coronal rain is visible with plane-of-sky velocities comparable to the downflow velocity of the satellite $\left(90 \mathrm{~km} \mathrm{~s}^{-1}\right.$, indicated by the white line). However, these inflows do not seem to extend to the position of the satellite feature in the umbra but appear to end at the lightbridge, as identified by the vertical, blue lines in the time-distance plot and the corresponding marks on the path in the HMI continuum context image.

On the other hand, the ratio of intensities of the satellite lines with the same post-launch calibration has the lower value of $1.6 \pm 0.3$. The ratio of widths is $1.1 \pm 0.3$. Moreover, the Si IV satellite lines are clearly broader than their main components: the ratio of the FWHM of the satellite lines to the corresponding main component is $1.35 \pm 0.4$ (see also Fig. 3). This contrasts with the observation that the widths of the O IV satellite lines are approximately equal to those of their main components (ratio: $0.95 \pm 0.15$ ). Since the peak intensities of the Si IV and O IV satellite lines are comparable (see again Fig. 3), it is implausible to suggest that a bias due to a possible instrumental background or solar continuum can explain these measurements.

It would therefore be tempting to interpret the larger width of the Si IV satellite lines as an optical thickness effect. Following the approach of Doschek \& Feldman (2004), this increased width would imply an optical thickness close to unity or larger (see for instance their Fig. 3). An optical thickness close to unity in the Si IV $1394 \AA$ line, with densities of approximately $10^{10}-10^{11} \mathrm{~cm}^{-3}$, as inferred in the following Sect. 3.3.2, implies a geometrical thickness of at least a few hundred kilometres for the emitting plasma. Whether optical thickness effects alone can explain the broader Si IV satellite lines (as well as their ratio) needs to be verified by more detailed models.

\subsubsection{Electron density}

The inter-combination multiplet of O IV lines at $1397.20 \AA$, $1399.77 \AA$, $1401.16 \AA, 1404.81 \AA$, and $1407.39 \AA$ provides a well known set of density-sensitive pairs (e.g. Dwivedi \& Gupta 1992). The lines at 1397.20 $\AA$ and $1407.39 \AA$ are outside the spectral window of this data set however, while the line at $1404.81 \AA$ is blended with an S IV line. We thus consider the ratio between the O IV $1399.77 \AA$ and $1401.16 \AA$ lines as density diagnostics. We adopted the CHIANTI database of atomic data version 7.1.4 (Dere et al. 1997; Landi et al. 2013), assuming a Maxwellian distribution. It should be noted that Dudík et al. (2014) have recently studied the effects of non-Maxwellian distributions of electron energies on both the Si IV and O IV multiplets. We feel, however, that it is unlikely the stationary flows that we are examining can sustain substantial departures from equilibrium energy distributions.

The time variation of densities inferred from this ratio is shown in Fig. 5, alongside the variation of the line intensities of both the main and the satellite components. To improve the signal-to-noise ratio, the line intensities were smoothed using a bi-Gaussian kernel with FWHM of three pixels along the slit and of five spectra in the time domain, corresponding to 
T. Straus et al.: A steady-state supersonic downflow in the TR above a sunspot umbra

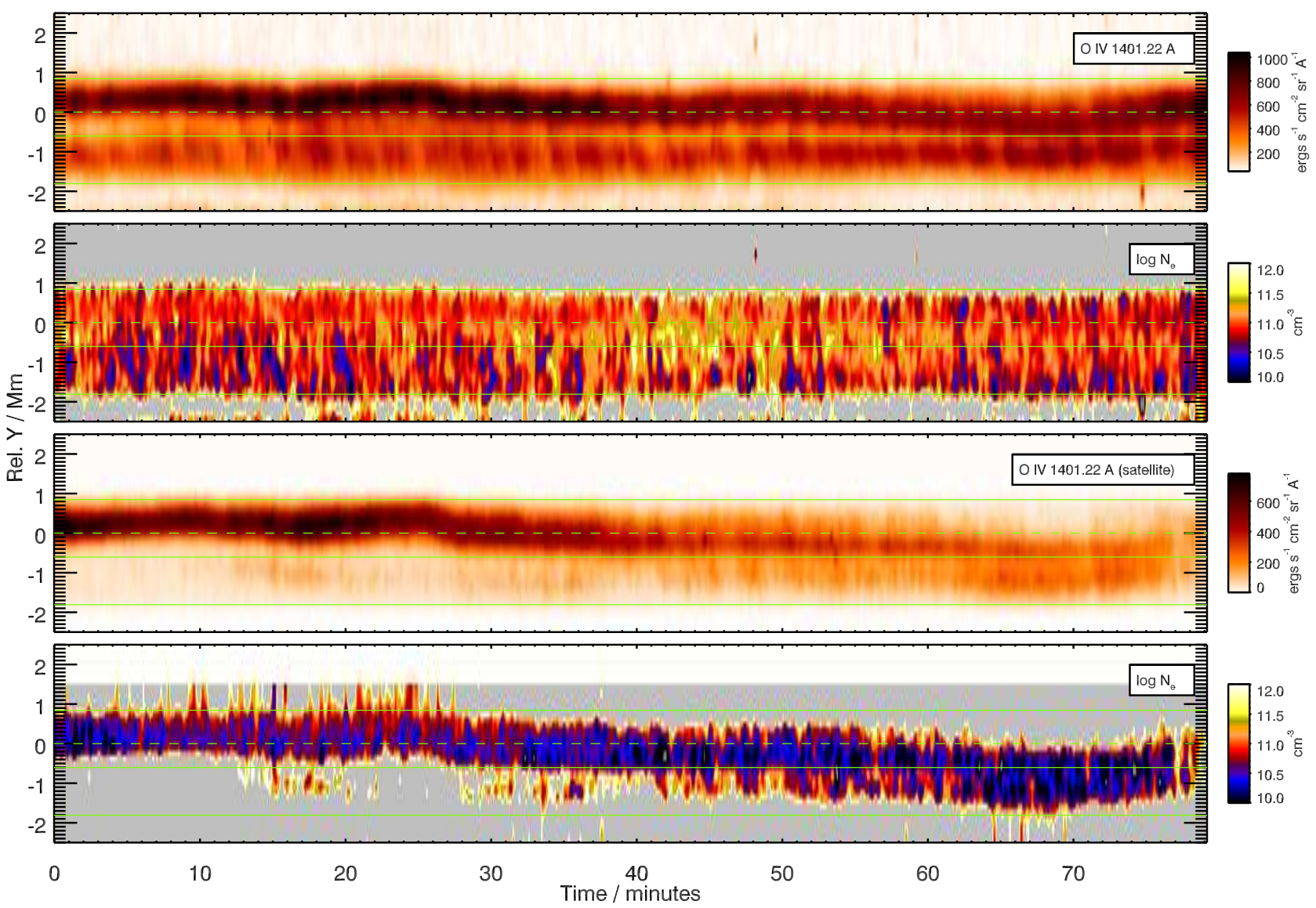

Fig. 5. Variation in the intensity of the main component of the O IV $1401 \AA$ line in the region that showed a supersonic downflow (first panel from the top), together with density inferred from the ratio of the O IV $1400 \AA$ and $1401 \AA$ lines (second panel). The variation of the intensity of the satellite line at $1401 \AA$ (third panel) and the density from the ratio of the satellite lines at $1400 \AA$ and $1401 \AA$ are shown in the third and fourth panels, respectively. The horizontal lines mark the boundaries of the regions given in Fig. 1, where the dashed line indicates Point C.

$0.35 \mathrm{Mm}$ and $15 \mathrm{~s}$, respectively. Smoothing data does not resolve the possibility of errors in determining the background continuum, which has to be subtracted from the line intensities before computing the line ratio. Since the O IV $1400 \AA$ line intensity is on average about a quarter of the intensity of the O IV $1401 \AA$ line in the region showing the supersonic downflow, a systematic error in the background correction will affect the former line more than the latter. The result would be a systematic bias in determinining the density. For instance, a residual background intensity in the O IV lines would explain why the line ratio, hence the density, appears to increase in those regions where the lines are weaker (e.g. at the edge of the region where the satellite lines are visible).

We examined the statistical distribution of densities derived from the ratio of the main components of the two O IV lines for the full spatial and temporal range over which the downflow is observable in this data set, i.e. for the full $80 \mathrm{~min}$ of the observing run and within $2.5 \mathrm{Mm}$ of Point $\mathrm{C}$ (Fig. 5). We obtained a mean value of $\log N_{\mathrm{e}}=10.95 \pm 0.20$ ( $1 \sigma$ uncertainty, $N_{\mathrm{e}}$ in $\mathrm{cm}^{-3}$ ), while the densities from the satellite lines are $\log N_{\mathrm{e}}=10.6 \pm 0.25$.

\subsection{Signatures of shock dynamics}

The temporal fluctuations of the Si IV and O IV lines show their main components with prominent shock-wave-passing maneuvres and rather stationary supersonic downflow satellite lines.
The satellite lines are not participating in the shock wave maneuvres, but in both lines the satellite intensity is weakly linked to the Doppler shift of the main component, showing a slightly brighter satellite feature when the main component passes through zero velocity during the steep rise in the shock front from maximum redshift to blueshift (Figs. 4 and 6). This suggests that the plasma of the downflow is, to a first approximation, dynamically decoupled from the plasma that forms the main components. One could speculate about a plume of plasma at transition region temperature above the actual transition region. This would be a different scenario from the cartoon presented in Fig. 14 of Nicolas et al. (1982). However, we detect subtle signs of a connection between the main and redshifted component, as we discuss below.

In Fig. 6 the main line parameters, determined as described in Sect. 3.2, are shown after averaging over all 13 pixels in the northern part of the downflow region. The shock-like temporal fluctuations are striking in both the Doppler shift (black curve in upper right panel) and main peak intensity (red curve), especially in the middle of the displayed part of the observations. The green crosses mark the zero-crossing times of velocity in the shock front passages. At those times, the main peak intensity is near its minimum.

The symbols in the lower right-hand panel of Fig. 6 show the intensity of the Si IV $1403 \AA$ redshifted satellite as a function of time. Here we averaged the satellite peak intensities over five consecutive measurements to reduce the noise. One can see 

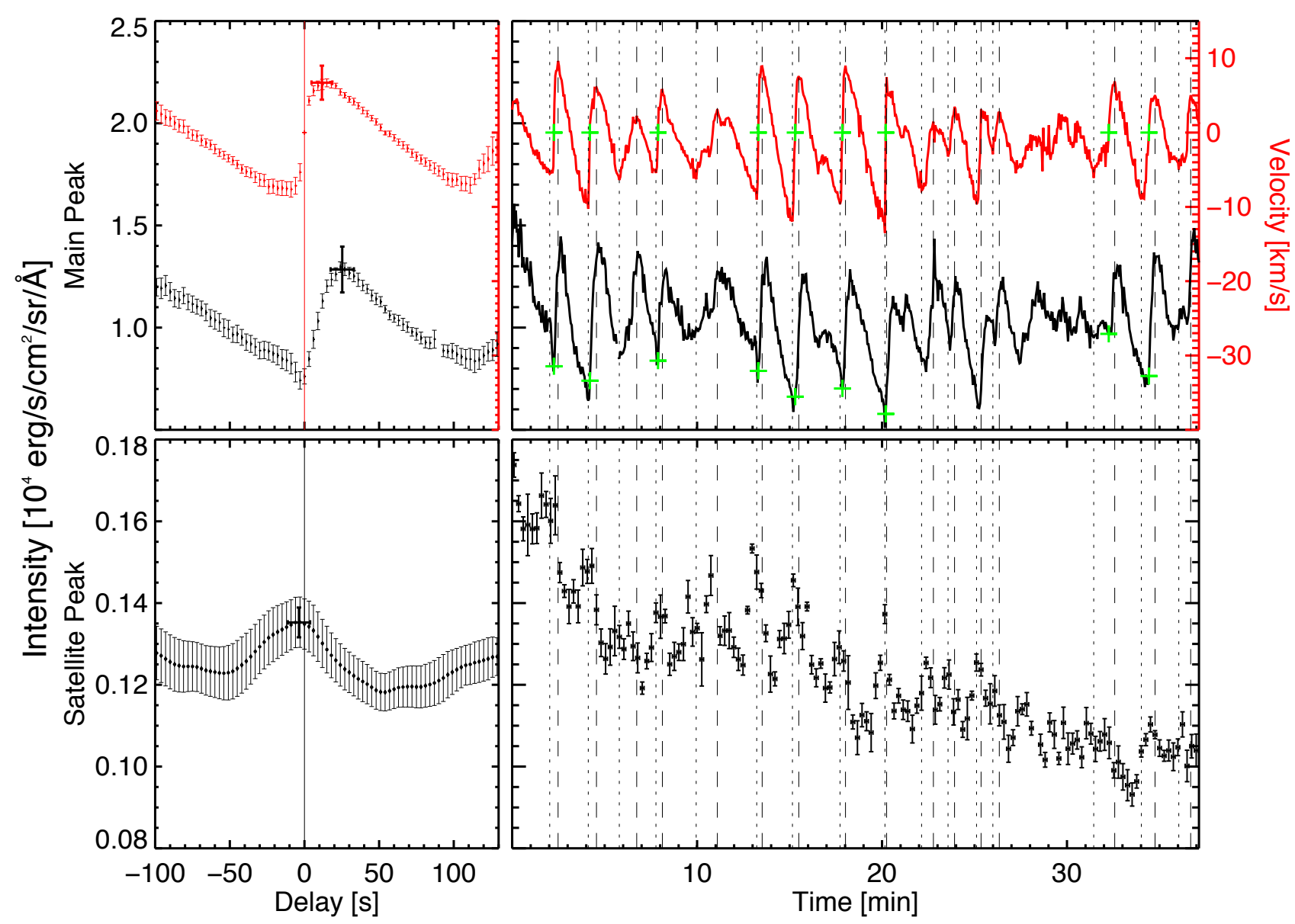

Fig. 6. Right panels: temporal evolution of Si IV line parameters averaged over the region where the line shows a satellite (black: intensity; red: velocity). Dotted and dashed vertical lines show the beginning and end of shock front passages, respectively. Positive velocities correspond to blueshifts. The times of zero velocity in the shock fronts are marked by green crosses. The main component's peak intensity shows a minimum at these times. The lower right panel shows the temporal evolution of the satellite's peak intensity. Left panels: temporal evolution of the average shock front of the nine shocks indicated by crosses in the upper right panel; time is given relative to the zero-crossing times of the main component's Doppler shift. The intensities of the Si IV satellite and main component are anti-correlated.

that the shock waves leave a weak imprint on the satellite intensity. However, there is an anti-correlation to the intensity of the main component. The satellite's maximum intensity is found at times of mimimum intensity in the main component (see black symbols in the left-hand panels of Fig. 6 that show the fluctuations averaged over the nine shock front events, with their zerovelocity marked by crosses in the upper right-hand panel, using the time of vanishing velocity as temporal reference). The error bars in the left-hand panels show the error of the mean value of the displayed signal after averaging over the nine shock events.

Interestingly, the satellite intensity (black symbols in the lower left-hand panel of Fig. 6) is not only anti-correlated to the intensity of the main component, but also shows a different shape. The shock-like shape is much less pronounced in the satellite's intensity profile, and it reveals a slight preference for the opposite sign having a slow rise before and a faster decrease after the peak intensity. This might hint at a downward shock. We come back to this particular feature below.

\section{Discussion}

The transition region is highly structured and dynamic (e.g. Mariska 1992). This fact has become even more obvious in recent high-resolution IRIS observations (e.g. Tian et al. 2014b; Hansteen et al. 2014). It is therefore quite remarkable that the downflow we observe in a sunspot umbra is rather stationary for nearly $1.5 \mathrm{~h}$. Has such a phenomenon been observed before? We believe that the one snapshot obtained during the HRTS I flight by Dere (1982) and Nicolas et al. (1982) and the SUMER observations of dual flows in $\mathrm{O} v$ and $\mathrm{N} \mathrm{V}$ in sunspots by Brynildsen et al. $(2001,2004)$ show the same phenomenon, albeit at a different (higher) temperature in the latter cases $(\mathrm{O} \mathrm{V}$ and $\mathrm{N} \mathrm{V}$ vs. Si IV and O IV).

What could be the origin of the feature we have seen? An obvious explanation is, of course, coronal rain, a commonly observed phenomenon, in the EUV and even $\mathrm{H} \alpha$ (e.g. Wiik et al. 1996; Schrijver 2001; de Groof et al. 2005; Müller et al. 2005a). To obtain information about flows at other temperatures, we inspected co-spatial and co-temporal SDO/AIA data and found material flowing towards the sunspot in all channels. In the right-hand panel of Fig. 4 we show the intensity in the $304 \AA$ passband, integrated across the path marked by the green line in the AIA $304 \AA$ context image, as a function of the distance from Point C. We find plane-of-sky inflows towards the sunspot reaching velocities of the order of the (vertical) downflow observed by IRIS (indicated by the continuous line of $90 \mathrm{~km} \mathrm{~s}^{-1}$ ). One could speculate that these flows feed the downflow plume. However, the signature of the inflowing material in the AIA channels disappears before reaching the lightbridge that is marked by the blue dashed lines in the time-distance plot and 


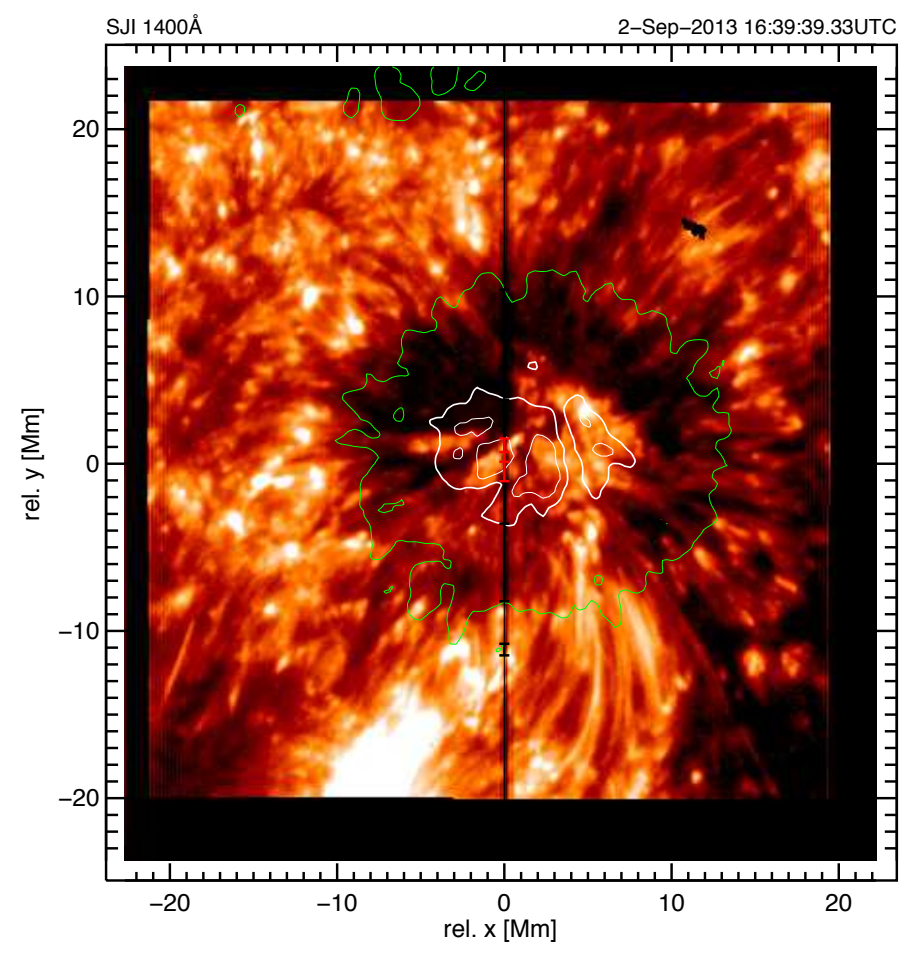

Fig. 7. IRIS $1400 \AA$ slit jaw image of the same area of AR 11836 as the HMI image in the lower left panel of Fig. 1. A time series of images is available online as a movie.

by the corresponding tick marks in the AIA $304 \AA$ context image in Fig. 4, leaving the central part of the umbra dominated by the umbral oscillations. These umbral oscillations are particularly strong in the $304 \AA, 171 \AA$, $211 \AA$ passbands and seem to originate in the centre of the umbra where the satellite feature is located, as already mentioned above.

In addition, we inspected the IRIS $1400 \AA$ slit-jaw data (see movie attached to Fig. 7), which also shows flows towards the sunspot. Again, as seen in the AIA $304 \AA$ data, the flows become invisible at the outer boundary of the penumbra. No flows or loops can be detected that connect to the core of the umbra.

Since none of these flows apparently connects to the centre of the sunspot umbra, where we observe the downflow in the transition region, we cannot ascertain a link between the supersonic transition region downflow, measured with the IRIS spectrograph, and the inflows towards the spot observed with AIA and the IRIS slit-jaw cameras. Moreover, as in most observations of coronal rain, we could only track individual "blobs" falling along the magnetic field lines. These blobs, if they really fell through to the satellite region around Point $\mathrm{C}$, would appear as strongly intermittent events. The intermittency of coronal rain is also a key characteristic of models of coronal rain (Müller et al. 2003, 2004, 2005b). Furthermore, coronal rain is often visible in chromospheric lines such as $\mathrm{H} \alpha$ and $\mathrm{Ca}$ II, whereas the downflow we observe is visible only in transition region lines formed around $10^{5} \mathrm{~K}$, with no trace of velocities in the cooler $\mathrm{C}_{\mathrm{II}}$ and $\mathrm{Mg}$ II lines. We therefore believe that coronal rain is not consistent with most of the observational facts listed in Sect. 3, but we cannot exclude it with certainty.

Another possible explanation, which we find more appealing, particularly when considering the remarkable stability of the supersonic downflow, is a siphon flow, as discussed by, for example, Cargill \& Priest (1980) and Noci (1981), who studied stationary flow solutions in a coronal magnetic arch. Below, we refer to Noci's paper, since it makes predictions that are more relevant to our observations. His "class (vii)" solutions are of particular interest here. These solutions describe subsonicsupersonic steady flows with stationary shocks that adjust the flow to the boundary conditions in the second foot point. The location and strength of the stationary shock is determined by the pressure ratio between the two foot points. With decreasing pressure in the downflow foot point, the shock moves closer towards the foot point, which results in increasing jumps in temperature and density. We should point out, though, that Noci's solutions are for coronal loops at coronal temperatures ( $>1 \mathrm{MK})$, whereas we observe the flow at transition region temperatures. However, we see no reason for these solutions to be limited to coronal temperatures. So we speculate that the supersonic downflow we see at transition region temperatures is the sign of a stationary shock in a siphon flow close to the foot point in the sunspot umbra.

In this scenario, the main component, which is dominated by the umbral 3 min shock waves, is formed in the part of the transition region underneath the stationary shock of the siphon flow. As mentioned above, the strength of the stationary shock in Noci's solutions depends on the pressure ratio between the foot points. Owing to the strong non-linear umbral 3 min oscillations, a constant pressure ratio between the two foot-points cannot be expected; however, a modulation of the stationary shock intensity by the underlying umbral 3 min shock waves might be. At minimum intensity in the main component, which coincides with minimum pressure and minimum temperature at the base of the downflow foot point, Noci's solutions predict the strongest stationary shock in the siphon flow. This is exactly what we observe. As seen in the lower left-hand panel of Fig. 6, the maximum intensity of the supersonic downflow coincides with minimum intensity of the main component.

Without having identified the upflow foot point, we are not in a position to conclusively claim the detection of a siphon flow at transition region temperatures and thus we can only speculate. Along these lines it is interesting to note that, about $21 \mathrm{~h}$ after the observations presented here, the AIA $171 \AA$ channel shows prominent coronal loops connecting the dark quarter of the sunspot umbra directly to the trailing plage of opposite polarity (Fig. 8). Could there have been a similar loop at cooler, transition region temperatures during our observations?

For some further insight into the evolution and extent of the downflow, we inspected all IRIS observations of AR 11836 on 2015 September 2 and 3. In addition to the data set studied in detail in this paper, there are seven more data sets of AR 11836. These consist of five large coarse 64-step rasters with a $2^{\prime \prime}$ step width and two two-step sparse rasters. One of these data sets, the large coarse raster starting on 2013-09-02T11:56:35 clearly shows signatures of a supersonic downflow for four steps (\#35-38) during all five raster scans. However, this downflow occurs at a different location in the sunspot, extending from the southern part of the most prominent lightbridge visible in Fig. 1 ( $x \sim 4 \mathrm{Mm}, y \sim-2 \mathrm{Mm}$ ) into the penumbra to the west. The measured downflow speed is lower $\left(\sim 75 \mathrm{~km} \mathrm{~s}^{-1}\right)$. Since there are only five scans (with a scan cycle time of $188 \mathrm{~s}$ ), it is difficult to infer information about temporal variations and their relation to the shock-wave-dominated main component. All we can say is that the feature lasted for more than $12 \mathrm{~min}$, has a similar extent along the slit as the downflow studied here, and is extended over at least $8^{\prime \prime}$ in the direction perpendicular to the slit. The large coarse raster scan following the observations studied in this paper (starting at 18:29:35 UT) also shows some hints of a downflow, but with a much weaker signal and not in consecutive frames, as if it had split up. None of the other four data sets 


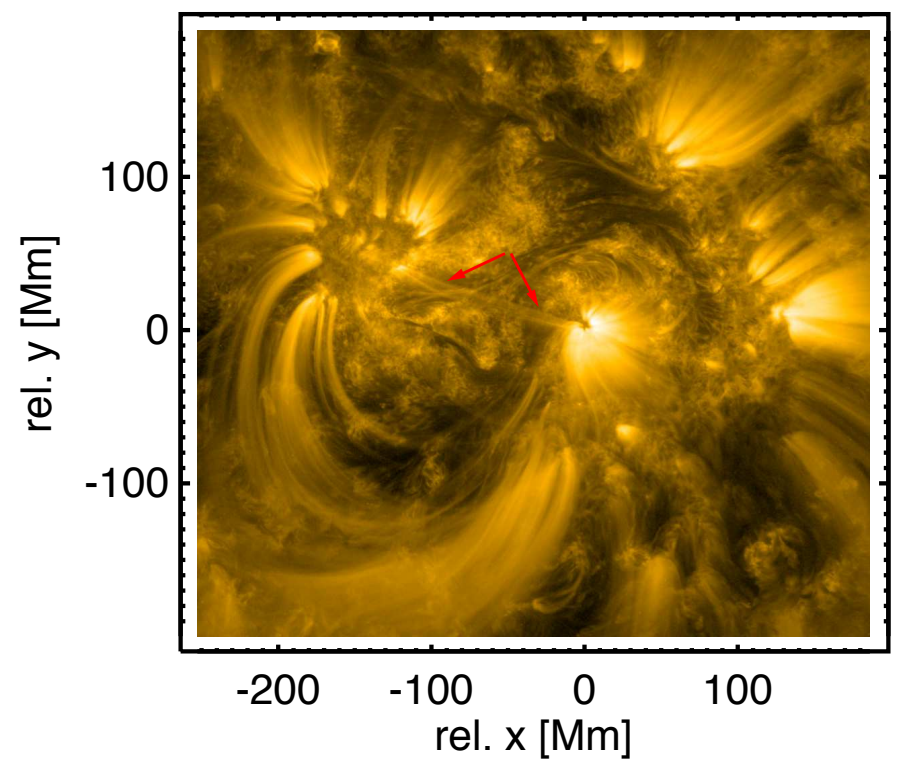

Fig. 8. AIA $171 \AA$ image of the same area as in Fig. 1, taken a day later on 2013 Sep. 3 14:20 UT. The coronal loops are indicated by red arrows, connecting the dark quadrant of the spot directly to the trailing plage of opposite polarity.

from September 2-3 shows any signs of a supersonic downflow. In a future paper, in which we will study the range of main properties, locations and rate of occurrence of this phenomenon, we will analyse these data sets in more detail.

From the measured density (see Sect. 3.3.2) of the downflow and its speed, $v \sim 90 \mathrm{~km} \mathrm{~s}^{-1}$, we can estimate the mass flux as $\rho v=N_{\mathrm{e}} m_{\mathrm{p}}\left(N_{\mathrm{H}} / N_{\mathrm{e}}\right) v$, where $\rho$ is the mass density and $m_{\mathrm{p}}$ is the proton mass, which yields $\sim 5 \times 10^{-7} \mathrm{~g} \mathrm{~cm}^{-2} \mathrm{~s}^{-1}$ assuming $N_{\mathrm{H}} / N_{\mathrm{e}}=0.83$. As such, this is a substantial flux. It would evacuate the overlying corona on time scales of the order of $10 \mathrm{~s}$. Even a prominence (column number density of $1 \times 10^{19} \mathrm{~cm}^{-2}$ ) would be drained in about $40 \mathrm{~s}$. For interpreting the flow as a siphon flow, such a high mass flux does not pose any problem.

\section{Conclusions}

We have observed a small-scale, supersonic downflow of about $90 \mathrm{~km} \mathrm{~s}^{-1}$ in the transition region above the sunspot umbra of AR 11836. The downflow is not visible in the chromospheric lines, which only show an intensity enhancement at the location of the downflow. The downflow shows up as redshifted, well-separated satellite lines of the Si IV and O IV transition region lines and is remarkably steady over the observing period of nearly $80 \mathrm{~min}$. The satellite lines do not participate in the 3 min shock wave Doppler maneuvres of the main component, but show a weak anti-correlated signature of the 3 min oscillations in intensity. We estimate the mass flux in the stationary flow to be $\sim 5 \times 10^{-7} \mathrm{~g} \mathrm{~cm}^{-2} \mathrm{~s}^{-1}$. This mass flux is relevant for the atmospheric structure, because it would evacuate an overlying quiet coronal column in about $10 \mathrm{~s}$.

We interpret these findings as evidence of a stationary termination shock of a supersonic siphon flow in a cool loop rooted in the central umbra of the spot, and we speculate that the supersonic flows seen by Dere (1982), Nicolas et al. (1982), and Brynildsen et al. $(2001,2004)$ are caused by the same phenomenon. We surmise that the 3-min shock waves, which are visible in the main component of the lines, form in the transition region underneath the stationary shock and represent a perturbation to the boundary condition of the stationary flow. This explains the anti-correlation between the intensity of the main and the redshifted satellite components.

Models of these supersonic siphon flows with termination shocks were developed by Cargill \& Priest (1980) and Noci (1981) for hot coronal loops, although they should be applicable to transition region temperatures as well. There are numerical studies of siphon flows in coronal loops (e.g. Mariska 1988; Spadaro et al. 1991; Orlando et al. 1995a), some including shocks (e.g. Orlando et al. 1995b), but we are not aware of any numerical studies that reproduce shocks of the magnitude we observe at transition region temperatures. Numerical studies of flows in the transition region (e.g. Antiochos 1984; Peter et al. 2004, 2006; Hansteen et al. 2010) usually aim to reproduce the average redshifts observed at those temperatures, and thus do not predict strong flows and shocks. It would therefore be interesting to consider numerical studies of solutions of the class studied by Noci, but for cool loops that never reach coronal temperatures.

There are numerous observations of flows in the photosphere, chromosphere, and corona that have been interpreted as siphon flows (e.g. Rueedi et al. 1992; Spadaro et al. 2000; Uitenbroek et al. 2006; Beck et al. 2010; Bethge et al. 2012). What are their relations, if any, to the supersonic downflows measured in the transition region over sunspot umbrae? Observations should also clarify whether steady-state supersonic downflows in the transition region are a common phenomenon that is visible only in certain circumstances or whether they are a rather rare phenomenon. If they are a regularly occurring phenomenon, what is the range of their main properties? We find it intriguing that the events with a single, clearly separated redshifted component, such as those observed by Dere (1982), Nicolas et al. (1982), one of the events of Brynildsen et al. (2001), and this work, have approximately the same speed $\left(\sim 90 \mathrm{~km} \mathrm{~s}^{-1}\right)$, which is at the high end of the average downflow speeds measured for five sunspots by Brynildsen et al. (2004).

Acknowledgements. IRIS is a NASA small explorer mission developed and operated by LMSAL with mission operations executed at NASA Ames Research Center and major contributions to downlink communications funded by ESA and the Norwegian Space Center (NSC). CHIANTI is a collaborative project involving George Mason University, the University of Michigan (USA), and the University of Cambridge (UK). We gratefully acknowledge helpful discussions with Bart De Pontieu, Joe Gurman, Jim Klimchuk, Daniele Spadaro, and Han Uitenbroek. This work has also benefited from discussions at the International Space Science Institute (ISSI) meeting, Heating of the magnetized chromosphere, from 5-8 January, 2015, where many aspects of this paper were discussed with other colleagues. We also thank an anonymous referee for useful comments.

\section{References}

Antiochos, S. K. 1984, ApJ, 280, 416

Avrett, E. H., \& Loeser, R. 2008, ApJS, 175, 229

Bartoe, J.-D. F., Brueckner, G. E., Nicolas, K. R., et al. 1979, MNRAS, 187, 463 Beck, C., Tritschler, A., \& Wöger, F. 2010, Astron. Nachr., 331, 574

Bethge, C., Beck, C., Peter, H., \& Lagg, A. 2012, A\&A, 537, A130

Borrero, J. M., \& Ichimoto, K. 2011, Liv. Rev. Sol. Phys., 8, 4

Brekke, P., Kjeldseth-Moe, O., \& Brueckner, G. E. 1990, Ap\&SS, 170, 135

Brekke, P., Brynildsen, N., Kjeldseth-Moe, O., Maltby, P., \& Bartoe, J.-D. F. 1991a, Adv. Space Res., 11, 251

Brekke, P., Kjeldseth-Moe, O., Bartoe, J.-D. F., \& Brueckner, G. E. 1991b, ApJS, 75,1337

Brosius, J. W., \& Landi, E. 2005, ApJ, 632, 1196

Brynildsen, N., Maltby, P., Brekke, P., et al. 1998, ApJ, 502, L85

Brynildsen, N., Maltby, P., Kjeldseth-Moe, O., \& Wilhelm, K. 2001, ApJ, 552, L77

Brynildsen, N., Maltby, P., Kjeldseth-Moe, O., \& Wilhelm, K. 2004, ApJ, 612, 1193 
T. Straus et al.: A steady-state supersonic downflow in the TR above a sunspot umbra

Cargill, P. J., \& Priest, E. R. 1980, Sol. Phys., 65, 251

de Groof, A., Bastiaensen, C., Müller, D. A. N., Berghmans, D., \& Poedts, S. 2005, A\&A, 443, 319

De Pontieu, B., Title, A. M., Lemen, J. R., et al. 2014, Sol. Phys., 289, 2733

Dere, K. P. 1982, Sol. Phys., 77, 77

Dere, K. P., Landi, E., Mason, H. E., Monsignori Fossi, B. C., \& Young, P. R. 1997, A\&AS, 125, 149

Doschek, G. A., \& Feldman, U. 2004, ApJ, 600, 1061

Dudík, J., Del Zanna, G., Dzifčáková, E., Mason, H. E., \& Golub, L. 2014, ApJ, 780, L12

Dwivedi, B. N., \& Gupta, A. K. 1992, Sol. Phys., 138, 283

Gurman, J. B. 1993, ApJ, 412, 865

Hale, G. E. 1908, ApJ, 28, 315

Hansteen, V. H., Hara, H., De Pontieu, B., \& Carlsson, M. 2010, ApJ, 718, 1070

Hansteen, V., De Pontieu, B., Carlsson, M., et al. 2014, Science, 346, 1255757

Jordan, C., Brueckner, G. E., Bartoe, J.-D. F., Sandlin, G. D., \& Vanhoosier, M. E. 1978, ApJ, 226, 687

Judge, P. G., Carlsson, M., \& Stein, R. F. 2003, ApJ, 597, 1158

Kjeldseth-Moe, O., Brynildsen, N., Brekke, P., et al. 1988, ApJ, 334, 1066

Kjeldseth-Moe, O., Brynildsen, N., Brekke, P., Maltby, P., \& Brueckner, G. E. 1993, Sol. Phys., 145, 257

Kleint, L., Antolin, P., Tian, H., et al. 2014, ApJ, 789, L42

Landi, E., Young, P. R., Dere, K. P., Del Zanna, G., \& Mason, H. E. 2013, ApJ, 763, 86

Lemen, J. R., Title, A. M., Akin, D. J., et al. 2012, Sol. Phys., 275, 17

Mariska, J. T. 1988, ApJ, 334, 489

Mariska, J. T. 1992, The solar transition region, Cambridge Astrophysics Series, 23
Müller, D. A. N., Hansteen, V. H., \& Peter, H. 2003, A\&A, 411, 605 Müller, D. A. N., Peter, H., \& Hansteen, V. H. 2004, A\&A, 424, 289 Müller, D. A. N., de Groof, A., de Pontieu, B., \& Hansteen, V. H. 2005a, 600, 30 Müller, D. A. N., De Groof, A., Hansteen, V. H., \& Peter, H. 2005b, A\&A, 436 , 1067

Nicolas, K. R., Bartoe, J.-D. F., Brueckner, G. E., \& Kjeldseth-Moe, O. 1982, Sol. Phys., 81, 253

Noci, G. 1981, Sol. Phys., 69, 63

Orlando, S., Peres, G., \& Serio, S. 1995a, A\&A, 294, 861

Orlando, S., Peres, G., \& Serio, S. 1995b, A\&A, 300, 549

Pesnell, W. D., Thompson, B. J., \& Chamberlin, P. C. 2012, Sol. Phys., 275, 3

Peter, H., Gudiksen, B. V., \& Nordlund, Å. 2004, ApJ, 617, L85

Peter, H., Gudiksen, B. V., \& Nordlund, A. 2006, ApJ, 638, 1086

Rempel, M., \& Schlichenmaier, R. 2011, Liv. Rev. Sol. Phys., 8, 3

Roussel-Dupre, R., Francis, M. H., \& Billings, D. E. 1979, MNRAS, 187, 9

Rueedi, I., Solanki, S. K., \& Rabin, D. 1992, A\&A, 261, L21

Scherrer, P. H., Schou, J., Bush, R. I., et al. 2012, Sol. Phys., 275, 207

Schrijver, C. J. 2001, Sol. Phys., 198, 325

Spadaro, D., Antiochos, S. K., \& Mariska, J. T. 1991, ApJ, 382, 338

Spadaro, D., Lanzafame, A. C., Consoli, L., et al. 2000, A\&A, 359, 716

Tian, H., Curdt, W., Teriaca, L., Landi, E., \& Marsch, E. 2009, A\&A, 505, 307

Tian, H., DeLuca, E., Reeves, K. K., et al. 2014a, ApJ, 786, 137

Tian, H., DeLuca, E. E., Cranmer, S. R., et al. 2014b, Science, 346, 125571

Uitenbroek, H., Balasubramaniam, K. S., \& Tritschler, A. 2006, ApJ, 645, 776

Wiik, J. E., Schmieder, B., Heinzel, P., \& Roudier, T. 1996, Sol. Phys., 166, 89

Yurchyshyn, V., Abramenko, V., \& Kilcik, A. 2015, ApJ, 798, 136 